\title{
Cavity Modes Study in Hyperuniform Disordered Photonic Bandgap Materials
}

\author{
Sam Tsitrin ${ }^{1}$, Ying quan $\mathrm{He}^{1}$, Sam Hewatt ${ }^{1}$, Brian Leung ${ }^{1}$, Weining Man $^{1}$, Marian Florescu ${ }^{2}$, Paul Steinhardt ${ }^{3}$, \\ Salvatore Tor quato ${ }^{3}$, Paul Chaikin ${ }^{4}$, \\ 1. Department of Physics and Astronomy, San Francisco State University, San Francisco, CA 94132, USA \\ 2. Advanced Technology Institute and Departm ent of Physics, University of Surrey, Guildford, Surrey GU27XH, United Kingdom \\ 3, Department of Physics, Princeton University, Princeton, NJ 08544, USA \\ 4, Department of Physics, New York University, New York, NY 20012, USA \\ tsitrin@mail.sfsu.edu
}

\begin{abstract}
We introduce novel architecture for cavity design in an isotropic disordered photonic band gap material. We demonstrate that point-like defects can support localized modes with different symmetries and multiple resonant frequencies, useful for various applications.

(C)2012 Optical Society of America

OCIS codes: $130.5296,130.7408,160.5293,160.5298$
\end{abstract}

Since photonic band gap (PBG) materials were first introduced, optical cavities (point defects in PBG materials) have attracted extensive attention due to the ir ability to trap light of certain frequencies within extremely small mode volume. Better understanding and control of frequency, location, and symmetry order of cavity modes inside the PBG will have an important impact on designing of novel sensors, filters, lasers, optical switches, and optical circuits [1].

Recently, it has been predicted and observed that hyperuniform disordered dielectric structures, which do not possess long-range translational order like crystals do, can also have complete PBGs [2-4]. More importantly the structures and PBGs are isotropic, not limited to crystalline rotational symmetry, hence allowing novel and flexible architecture of cavity (point defects) and wave-guide (line defects) design.

According to recent simulation studies by Florescu et al, introducing a point defect by removing a single dielectric cylinder from a $2 \mathrm{D}$ hyperuniform PBG structure, results in a localized cavity mode with monopole symmetry[5]. The electric field oscillation pattern was predicted to extend 1-2 cell widths into the surrounding structure (Figure 1). When a defect dielectric cylinder of increasing radius is used to replace a regular one, the electric field of the localized cavity modes would oscillate with changing symmetries. For every symmetry order, such as monopole, dipole, quadrupole, hexapole and octopole, it was observed that an increase in defect cylinder radius will introduce higher resonant frequencies inside the $\mathrm{PBG}$ region.

Our experimental structure is assembled using $\mathrm{Al}_{2} \mathrm{O}_{3}$ cylindrical rods $(r=$ $2.5 \mathrm{~mm}, h=10 \mathrm{~cm}$ ), inserted into a platform of a hyperuniform disordered pattern with $1 \mathrm{~cm}$ deep slots. The average cell size (spacing between rods) is $a=13.3 \mathrm{~mm}$. The structure has a TM polarization PBG from 9.2 to 10.7 GHz. Cavities are easily generated and changed in this structure by removing rods to create voids and placing bundled clusters of rods into the voids. We use horn antennas attached to a microwave vector network

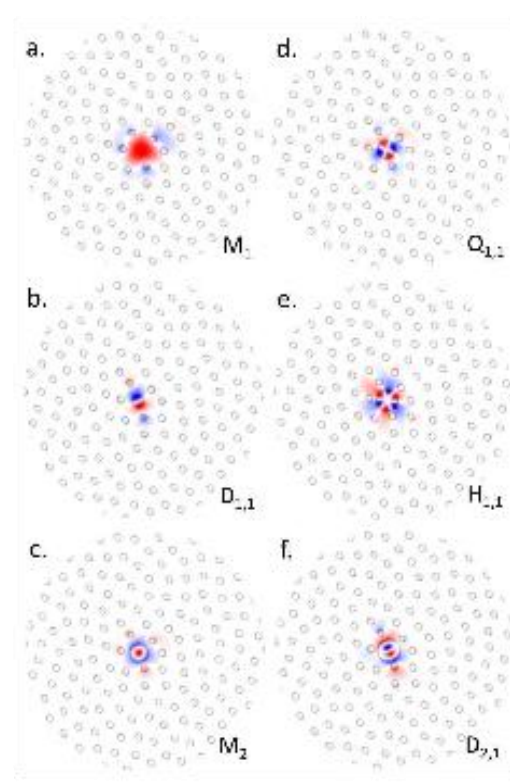

Figure 1. Electric field distribution for cavity modes of different symmetries obtained by changing the size of the defect.

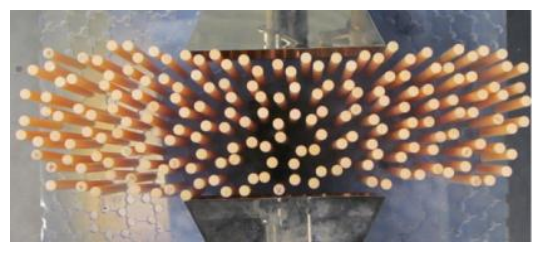

Figure 2. A photograph of the sample slice and experimental set up. 
analyzer to measure the reflection and transmission through a slice of the hyperuniform disordered structure, a few wavelengths thick, with and without those cavities (Figure 2).

As shown in Figure 3, cavity modes are revealed by large transmission peaks inside the PBG frequency region. By removing two cylinders in the middle of the structure, cavity modes around $10.3 \mathrm{GHz}$ are excited. The transmission peaks associated with those cavity modes decrease exponentially with the thickness of the sample slice, and still remain detectable when the cavities are at a distance of $2.5 a$ away from the sample edge.

To investigate the symmetry properties of the cavity modes, we introduced bundled clusters of alumina rods in different arrangements and orientations. As was predicted in the simulation study, an increase of the high-index dielectric defect radius will push the resonant frequency higher for a particular order of symmetry mode. As shown in Figure 4, introducing a 3-rod, 4-rod, and 7-rod cluster, respectively, resulted in various resonant peaks of different cavity modes.

The great flexibility in tuning these cavity modes in the HD structure, combined with its isotropy, makes it possible to guide and filter light of desired frequencies around arbitrary sharp bends. Figure 5 shows a photo of and transmission through a $50^{\circ}$ bend, which can be considered as two straight channels joined by a cavity at the corner. Waves of various frequencies inside the PBG are guided and transmitted through this sharp bend. The resonant frequencies in this cavity were modified and optimized by adding and removing various rods. This flexibility and abundance of cavity modes will be important for filtering and tuning applications.

In summary, we have experimentally demonstrated that sharp PBG resonant modes are attainable in a hyperuniform disordered structure, and we can tune

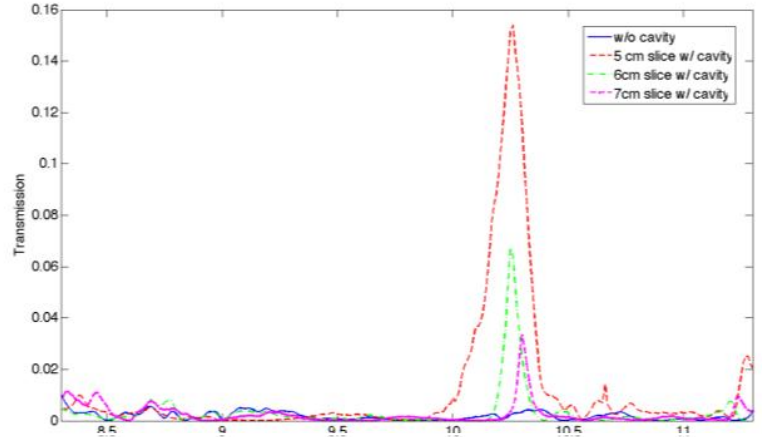

Figure 3. TM polarization transmismission through a cavity decorated HD struct ure slice of varying thickness.

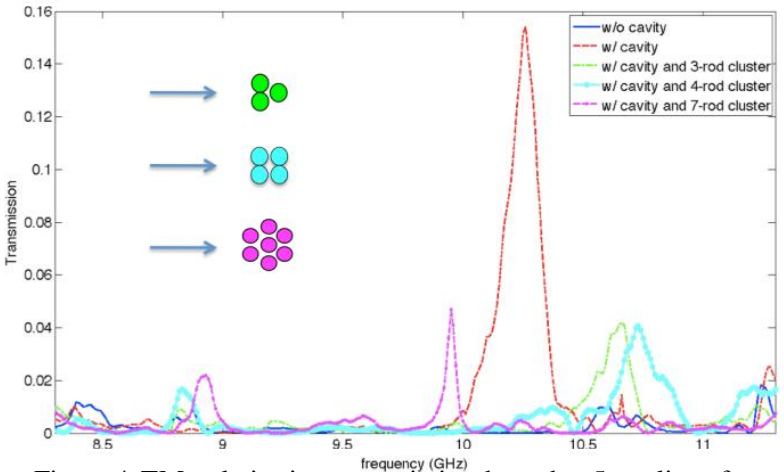

Figure 4.TM polarization transmission through a $5 \mathrm{~cm}$ slice of hyperuniform structure decorated with a cavity and various clusters
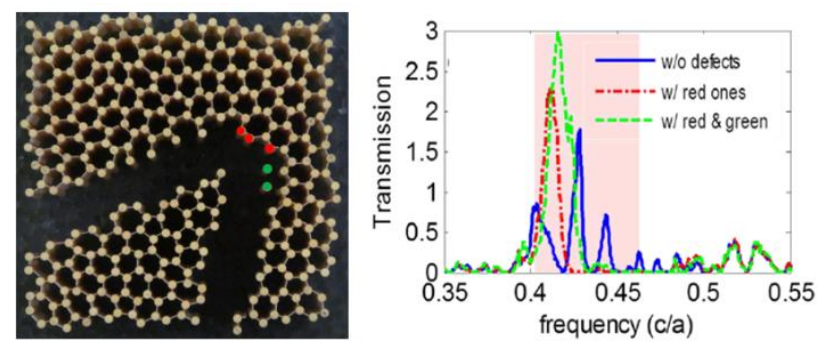

Figure 5.TM polarization transmission through a $50^{\circ}$ bend. Filtered frequencies can be easily tuned by modifying the cavity modes at the corner. PBG is highlighted with a pink strip. Transmission is normalized to the transmitted energy between two facing horns sepa rated by the total channel length in free space. the frequency of the modes by varying the dielectric defects inside the cavity as predicted by simulations. The ability to control, localize, and slow down EM waves ins ide solids will have a great impact on future technological development of optical switches, lasers and sensors. While this study currently uses horn antennas outside of the structure sample to detect resonance, our future investigation will focus on using dipole antennas inside the cavity for a more precise and quantitative study of the resonance phenomena.

This work was supported by the Research Cooperation and SFSU's startup fund.

[1] John D. Joannopoulos, etc. Photonic Crystals: Molding the Flow of Light (Princeton University Press, Princeton, 2008)

[2] Marian Florescu, etc. PNAS, 10649 20658-20663 (2009)

[3] Weining Man, etc. CLEO CThS2 (2010)

[4] Weining Man, etc. CLEO QF3H2 (2012)

[5] Marian Florescu, etc "Novel Optical Cavity Modes and Waveguide Geometries in Hyperuniform Disordered Photonic Solids", unpublished 